\title{
Research on Cloud Bus Architecture of Smart Grid
}

\author{
Xuewei Shang ${ }^{1,2}$, Lin Zhao ${ }^{1,2}$, Fei Ye ${ }^{1,2}$, Heng Wang ${ }^{1,2}$, Zelong Fan ${ }^{1,2, *}$, Guangmin Fan ${ }^{3}$, Zheng Mei ${ }^{1,2}$, Lingxu Guo ${ }^{3}$, \\ Qipeng $\mathrm{Li}^{1,2}$, Ziming $\mathrm{Guo}^{4}$, Yi Lư ${ }^{5}$, Liang $\mathrm{Guo}^{5}$ and Bo $\mathrm{Yan}^{4}$ \\ ${ }^{1}$ NARI Group Corporation (State Grid Electric Power Research Institute), Nanjing 211106, China \\ ${ }^{2}$ Beijing Kedong Electric Power Control System Co. Ltd., Beijing 100085, China \\ ${ }^{3}$ State Grid Tianjin Electric Power Company, Tianjin 300010, China \\ ${ }^{4}$ Hebei North Electric Co. Ltd., Beijing 100053, China \\ ${ }^{5}$ State Grid Sichuan Electric Power Company, Chengdu 610000, China \\ ${ }^{*}$ Corresponding author
}

\begin{abstract}
With the construction of UHV power grid, power grid operation gradually presents the integration characteristic. Hence, it is much more difficult for the current intelligent power grid dispatching and control system's bus to meet the needs of data exchange among the different dispatching agencies. According to the characteristics of the smart grid, a cloud bus architecture is proposed in this paper, while the power dispatching domain name, distributed proxy, cloud service management and access, cloud message management and transmission are studied in order to achieve sharing of services and efficient transmission of data and expound the prospect of cloud bus finally.
\end{abstract}

Keywords-cloud bus; distributed proxy; cloud service management and access; cloud message management and transmission

\section{INTRODUCTION}

In recent years, the AC-DC coupling relationship is further strengthened [1-2], and this brings a great challenge to the regulation and operation. The demand for multi-level dispatching mechanism integration scheduling is increasing. In order to ensure the safe and stable operation of the power grid, it is necessary to realize the same scene of power grid operation and monitoring, synchronous fault detection and synchronization, early warning of risk hidden danger. To this end, it is necessary to build a strong cloud data transmission system to ensure the transmission of large-scale real-time information. It can support large-scale integrated operation of the grid.

At present, the state grid corporation of China has built a smart grid dispatching control system covering the national, sub-center and province power dispatching and control centers, which realizes the service access and message transmission inside the dispatching and control center through the message bus and the service bus[3-4]. It plays a certain role for the safe and stable operation of the power grid. However, there are still some problems in the large amounts of message transmission and service access between the different dispatching and control centers: There is no message transfer mechanism can be used in real-time messages that need to be transmitted between different dispatching and control centers, such as grid state information and important alarm events; the lack of uniform and efficient service access mechanism between different dispatching and control centers. It is necessary to share the calculation and analysis of the whole network, integrated intelligent alarm, DSA calculation results and other service data in order to meet the requirements of integration and coordination operation; smart grid dispatch control system has a single remote proxy function, which cannot support real-time message transmission and large-scale service access in a cloud.

This paper proposes a cloud bus based on power dispatching data network. This bus can be used to form a unified and efficient cloud data transmission mechanism in dispatching and control centers. The bus is composed of key parts such as power dispatch domain name, distributed proxy, cloud service bus and cloud message bus. It realizes real-time and reliable data transmission in a cloud.

\section{Cloud Bus ARCHITECTURE}

Power dispatching data network is a dedicated data network for power dispatching services, which is mainly responsible for production control area business [5]. It has the characteristics of geographical distribution, large number of nodes, high realtime and reliability.

The cloud bus based on the power dispatching data network is the information highway which runs through the national, sub-center and so on multi-level dispatching and control system. It can support cross-regulation system data exchange, such as real-time data, historical data, model data and various application calculation results.

The structure is shown below.

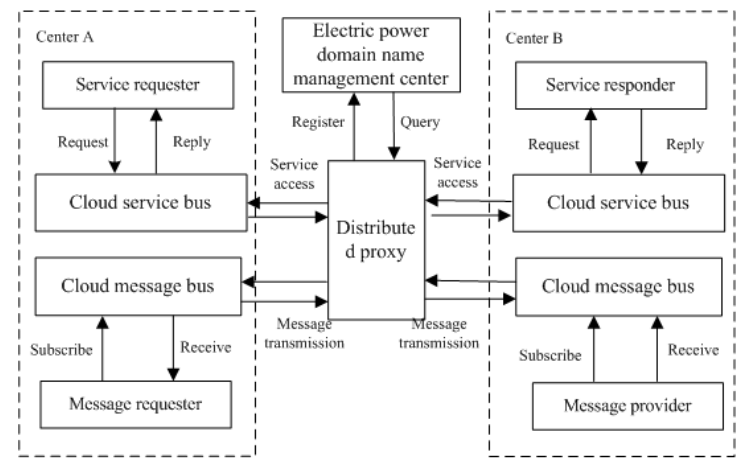

FIGURE I. CLOUD BUS ARCHITECTURE 
The power dispatch domain name is the foundation of the cloud bus architecture. Through the uniform naming mechanism of the different dispatching and control centers, the domain name naming mode of the multi-level control system is standardized and managed, and the domain name to the network address can be quickly analyzed and searched. The distributed proxy establishes the data transmission channel that transparent to the application. It provides the underlying data exchange function in the cloud bus, and the fast and efficient data communication support for the cloud service bus and cloud message bus. Cloud service bus has established a common framework for service access. Based on cloud service management and discovery technology and cloud service access technology, service access between different dispatching and control centers is realized. Through the underlying service registration and upper information synchronization, to achieve global sharing of service information, service inquiries and positioning. The cloud message bus establishes the message transmission framework. Through the cloud message subscription management technology and the cloud message transmission technology, the message transmission and management between the different dispatching and control centers are realized.

As a unified cloud data interaction system, the cloud bus has the following characteristics: (1) It is a highly efficient and real-time data transmission between different dispatching and control centers that can support large-scale concurrent data transmission. (2) It provides a common mechanism for service access mechanism to support the whole network sharing of service data. (3) Through the mechanism of message transmission across the different dispatching and control centers, can realize the message interaction in a cloud. (4)Reliable data transmission, through the cloud service and cloud message management to ensure that service access and message transmission's security and reliable.

\section{Key Technologies of Cloud Bus}

\section{A. Power Dispatch Domain Name}

At present, there is no independent power dispatch domain name in the smart grid dispatching control system, and there is only a domain name management function that highly coupled with the service proxy. In order to solve the problem of address routing between different dispatching and control centers, this paper proposes the domain name of power dispatching to support cloud dispatching service.

The power dispatch domain name refers to the naming mode of the Internet domain name. According to the characteristics of the dispatching and control center's name, the power dispatch domain name is combined by the top-down order. Organization name + Subordinate dispatch organization name, with a '.' In the middle. Sub-center C domain name is "Sub-center C". The provincial A is contained by sub-center C, its domain name called "Sub-center C. Provincial A".

The power domain name management center formed by using the power dispatch domain name adopts the method of registering the lower level to the superordinate level, registering each other at the same level and unified management. It provides the domain name's binding with the external communication address and the domain information's sharing between the different dispatching and control centers. At the same time, it can analysis of Addresses and Selection of Optimal Performance Nodes to different dispatching and control centers through domain routing. The power domain name management center operates in a hot standby mode. The host and backup node sense the online status of each other through the heartbeat. When the host failure or downtime, the backup node automatically take over the operation.

\section{B. Distributed Proxy Data Communication Technology}

In order to realize the large-scale data exchange between different levels of scheduling agencies and provide the infrastructure support for the global sharing of grid operation data, this paper proposes a distributed proxy data communication technology.

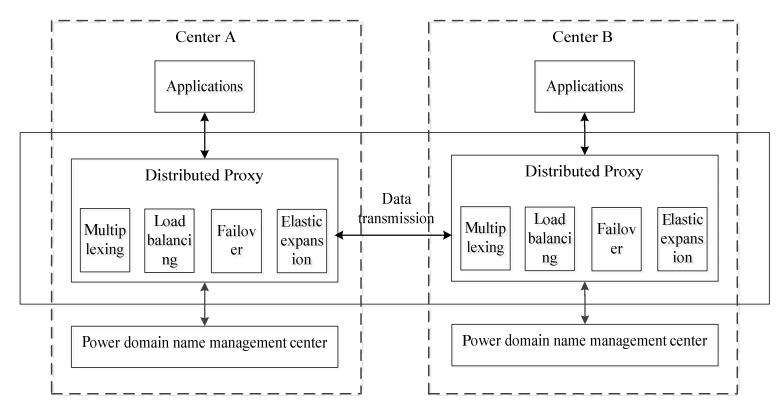

FIGURE II. DISTRIBUTED PROXY COMMUNICATION TECHNOLOGY SCHEMATIC DIAGRAM

As shown in Figure II, distributed proxy data communication technology includes link multiplexing, load balancing, failover and elastic expansion. Link multiplexing implements a variety of link management functions such as creation, reuse, and reclamation of connections between different dispatching and control centers. Load balancing provides the optimal access node to other distributed proxy clusters. The failover mechanism can retransmit data from other nodes in the distributed proxy cluster when forwarding data fails. The elastic extension is responsible for dynamically adding or deleting the distributed proxy node without affecting the other running nodes.

Distributed proxy data communication technology allows multiple types of data to be transferred between different dispatching and control centers.

\section{Cloud Service Management and Access Technology}

To ensure the stable operation of services in a cloud, this paper proposes a cloud service management technology to monitor and manage services in a unified manner. 


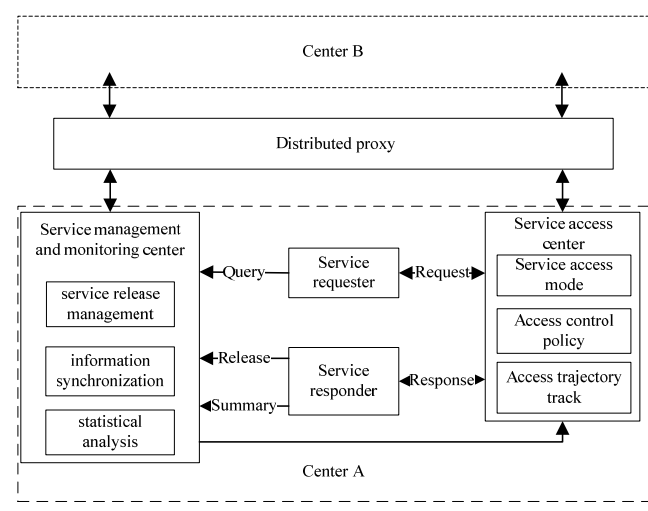

FIGURE III. CLOUD SERVICE BUS FRAMEWORK

As shown in Figure III, cloud service management technology contains cloud service release management, information synchronization, and statistical analysis. The cloud service release management is responsible for the application service's registration, deletion and invalidation detection. The service is registered to the local service management and monitoring center. The information synchronization is responsible for synchronizing the service information in the local service management and monitoring center to other dispatching and control centers in a direct or on-demand synchronization manner. The direct synchronization mode is used for synchronization between dispatching and control centers with direct upper and lower level relationships Information. The on-demand Synchronization mode is used for synchronization between dispatching and control centers that require multiple levels of traversal in the horizontal or vertical directions. The statistical analysis is responsible for real-time statistics the service running conditions, service load and service information trajectory and other information.

Through the cloud service management technology, it realizes the service synchronization awareness and improves the efficiency of the query and retrieval services among the different dispatching and control centers. The statistical analysis of the cloud service can timely alert the abnormal state and can help positioning and troubleshooting the problem.

As shown in Figure III, cloud service access technology includes service access mode, access control policy and access trajectory tracking mechanism. Service access is used to service information interaction between different dispatching and control centers. Combining the service characteristics of the smart grid dispatching control system and the existing application service access method, the service access mode of the master and backup and load balancing are designed. The visitor can choose the service access mode flexibly. In order to prevent the illegal users and their illegal access from the system, the cloud service management technology proposes a multigranularity access control strategy that contains regional access restrictions, digital certificate authentication and IP address control. Access trajectory tracking mechanism is the service access monitoring of whole process in a cloud. It tracks every aspect of the access process and provides convenient and accurate information for the location of abnormal events.
Through cloud service access technology, a universal and efficient cloud service access mechanism is formed, and the security and reliability of service access and access within a cloud are ensured.

\section{Cloud message Management and Transmission Technology}

In response to the need for efficient, reliable message transmission, a cloud message management technique is proposed in this section. The cloud message management technology is used to establish the cloud message subscription / distribution mechanism and to manage the cloud message subscription information. It can also monitor the running state of the cloud message bus and the transmission trajectory of the message.

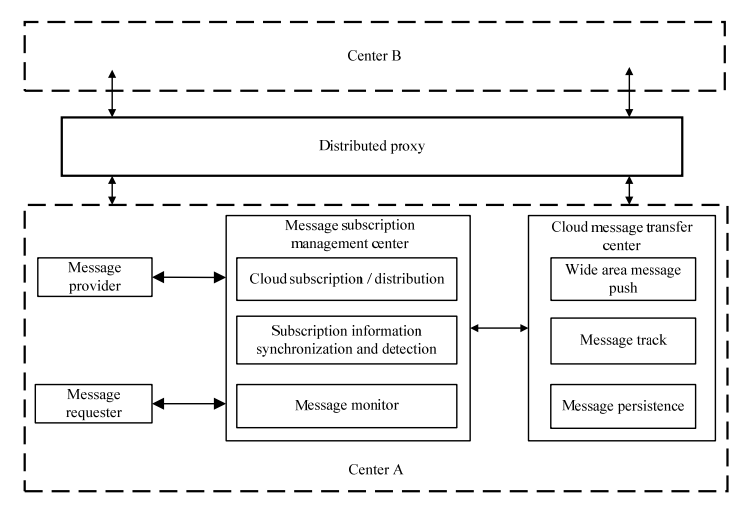

FIGURE IV. CLOUD MESSAGE BUS FRAMEWORK

As shown in Figure IV, cloud message management techniques includes cloud subscription / distribution mechanisms, cloud subscription information synchronization and detection, cloud message monitoring methods. The cloud subscription / distribution mechanism defines the composition of the cloud subscription information, and realizes the subscription process and storage of the subscription information.

The cloud subscription information synchronization and detection is used to realize the sharing of cloud message subscription information among distributed proxy cluster, and to detect the expired cloud message subscription information and clear it in time to avoid waste of system resources. The cloud message monitoring is used to monitor the health and statistics of the cloud message bus in real time. Monitoring content includes transmission link status, cloud message subscription information, number of messages sent and received in a fixed period (per second, per minute, etc.), message traffic size, etc.

The cloud messaging technology is used to provide realtime message forwarding between different dispatching and control centers. Applications can push messages efficiently and reliably to one or more dispatching and control centers to ensure the timeliness and orderly transmission of messages.

As shown in Figure IV, cloud message transmission technology includes cloud message push, tracking strategy, message persistence. The cloud message push can complete the 
message forwarding function by establishing the cloud message transmission center. The cloud message tracking strategy includes message aging management and source domain management. The aging management is to manage the life cycle of the cloud message to avoid the transmission of expired messages. The source domain management is to identify the cloud message sources to avoid the message loopback. The cloud message persistence is to ensure message transmission. It save the message to the disk file, send the message after the success of the file batch delete. If the network or program failure and other reasons to send messages failed, it use the link to reconnect and message breakpoints continued transmission and other means to ensure the reliable transmission of information.

\section{APPLiCATION PROSPECT}

Adopting cloud bus the dispatching and control system has been deployed and applied in several sub-centers, mainly used for cloud viewing of pictures and integrated intelligent alarm applications in the nation and sub-center's smart grid dispatching control system for message transmission. Through the promotion and application of this architecture, it can effectively improve the information exchange.

The cloud bus will improve the level of UHV power grid operation and monitoring and reduce the fault response time to ensure the UHV power grid safe and stable operation.

\section{CONCLUSION}

Aiming at the requirement of data interchange across various applications, and combining the characteristics of dispatching data network and the current bus in the smart grid dispatching control system, a cloud bus architecture based on dispatching data network is established. The architecture can be used to interconnect the control systems of the independent dispatching and control center, and implement the real-time synchronization and sharing of large-scale message and service information. The preliminary practice shows that this architecture can implement the message transmission and service sharing among the national, sub-centers and provincial control systems. With the cloud bus further popularization and application, it is hoped that real-time message transmission and service sharing will be carried out in the whole network in the future, which will help to solve the problem of integration of master station and substation, model data center, power application service and other control system data transmission problems.

\section{REFERENCES}

[1] ZHANG Dongxia, YAO Liangzhong, MA Wenyuan. "Development Strategies of Smart Grid in China and Abroad”. Proceedings of the CSEE, pp. 1-14, 2013(31).

[2] LI Wei, XIN Yaozhong, SHEN Guohui, HUANG Kun, CAO Rongrong, MENG Xin, WAN Shupeng. "Scheme of Power Grid Graphics Maintenance and Sharing Based on CIM/G”. Automation of Electric Power System, pp. 42-47, 2015(1).

[3] XIN Yaozhong, SHI Junjie, ZHOU Jingyang, GAO Zonghe, TAO Hongzhu, SHANG Xuewei, et al. "Technology Development Trends of Smart Grid Dispatching and Control Systems”, pp. 2-8, 2015,39(1).
[4] WANG Heng, XIN Yaozhong, SHANG Xuewei, YAN Yaqin, LI Qipeng, MU Haijun. "A New Data Bus Technology for Smart Grid Dispatching and Control Systems”, pp. 9-13, 2015,39(1).

[5] Hu Juan, Li Zhihuan, Duan Xianzhong. "Structural Feature Analysis of the Electric Power Dispatching Data Network, pp. 53-59, 2009,29(4). 